\title{
Effect of Dapagliflozin on Myocardial Insulin Sensitivity and Perfusion: Rationale and Design of The DAPAHEART Trial
}

\author{
Gian Pio Sorice (D) - Francesca Cinti (D) - Lucia Leccisotti (D) - Domenico D'Amario (D) • \\ Margherita Lorusso (D) - Maria Angela Guzzardi (D) - Teresa Mezza (D) - Camilla Cocchi (D) · \\ Umberto Capece (D) · Pietro Manuel Ferraro (D) - Filippo Crea (D) - Alessandro Giordano (D) • \\ Patricia Iozzo (D) - Andrea Giaccari (D
}

Received: April 9, 2021 / Accepted: May 12, 2021 / Published online: May 26, 2021

(C) The Author(s) 2021

\section{ABSTRACT}

Introduction: Sodium-glucose co-transporter-2 (SGLT-2) inhibitors have been shown to have beneficial effects on various cardiovascular (CV) outcomes in patients with type 2 diabetes (T2D) in primary prevention and in those with a high $\mathrm{CV}$ risk profile. However, the mechanism(s) re-

GianPio Sorice, Francesca Cinti and Lucia Leccisotti contributed equally to this work.

Supplementary Information The online version contains supplementary material available at https:// doi.org/10.1007/s13300-021-01083-1.

G. P. Sorice · F. Cinti · T. Mezza - C. Cocchi ·

U. Capece · A. Giaccari ( $\square)$

Dipartimento di Scienze Mediche e Chirurgiche,

Centro Malattie Endocrine e Metaboliche,

Fondazione Policlinico Universitario A. Gemelli

IRCCS-Università Cattolica del Sacro Cuore, Rome, Italy

e-mail: andrea.giaccari@unicatt.it

G. P. Sorice

Department of Emergency and Organ

Transplantation, Section of Internal Medicine,

Endocrinology, Andrology and Metabolic Diseases,

University of Bari Aldo Moro, Bari, Italy

L. Leccisotti · M. Lorusso · A. Giordano

Dipartimento di Diagnostica Per Immagini,

Radioterapia Oncologica ed Ematologia, UOC di

Medicina Nucleare, Fondazione Policlinico

Universitario A. Gemelli IRCCS-Università Cattolica

del Sacro Cuore, Rome, Italy sponsible for these $\mathrm{CV}$ benefits remain elusive and unexplained. The aim of the DAPAHEART study will be to demonstrate that treatment with SGLT-2 inhibitors is associated with greater myocardial insulin sensitivity in patients with $\mathrm{T} 2 \mathrm{D}$, and to determine whether this improvement can be attributed to a decrease in wholebody (and tissue-specific) insulin resistance and to increased myocardial perfusion and/or glucose uptake. We will also determine whether there is an appreciable degree of improvement in myocardial-wall conditions subtended by affected and non-affected coronary vessels, and if this relates to changes in left ventricular function.

\section{D'Amario · F. Crea}

Dipartimento di Scienze Cardiovascolari, UOC di Cardiologia, Fondazione Policlinico Universitario A. Gemelli IRCCS-Università Cattolica del Sacro Cuore, Rome, Italy

M. A. Guzzardi · P. Iozzo

Istituto di Fisiologia Clinica, Consiglio Nazionale Delle Ricerche (CNR), Pisa, Italy

\section{P. M. Ferraro}

Dipartimento di Scienze Mediche e Chirurgiche, UOC di Nefrologia, Fondazione Policlinico Universitario A. Gemelli IRCCS-Università Cattolica del Sacro Cuore, Rome, Italy 
Methods: The DAPAHEART trial will be a phase III, single-center, randomized, two-arm, parallel-group, double-blind, placebo-controlled study. A cohort of 52 T2D patients with stable coronary artery disease (without any previous history of myocardial infarction, with or without previous percutaneous coronary intervention), with suboptimal glycemic control (glycated hemoglobin [HbA1c] 7-8.5\%) on their current standard of care anti-hyperglycemic regimen, will be randomized in a $1: 1$ ratio to dapagliflozin or placebo. The primary outcome is to detect changes in myocardial glucose uptake from baseline to 4 weeks after treatment initiation. The main secondary outcome will be changes in myocardial blood flow, as measured by ${ }^{13} \mathrm{~N}$-ammonia positron emission tomography/computed tomography (PET/CT). Other outcomes include cardiac function, glucose uptake in skeletal muscle, adipose tissue, liver, brain and kidney, as assessed by fluorodeoxyglucose (FDG) PET-CT imaging during hyperinsulinemic-euglycemic clamp; pericardial, subcutaneous and visceral fat, and browning as observed on CT images during FDG PETCT studies; systemic insulin sensitivity, as assessed by hyperinsulinemic-euglycemic clamp, glycemic control, urinary glucose output; and microbiota modification.

Discussion: SGLT-2 inhibitors, in addition to their insulin-independent plasma glucose-lowering effect, are able to directly (substrate availability, fuel utilization, insulin sensitivity) as well as indirectly (cardiac after-load reduction, decreased risk factors for heart failure) affect myocardial functions. Our study will provide novel insights into how these drugs exert $\mathrm{CV}$ protection in a diabetic population.

Trial registration: EudraCT No. 2016-00361427; ClinicalTrials.gov Identifier: NCT03313752.

Keywords: Dapagliflozin; SGLT-2; Myocardial glucose uptake; Myocardial insulin sensitivity; Myocardial blood flow; Coronary flow reserve; PET; Myocardial dysfunction; Precision medicine

\section{Key Summary Points}

Type 2 diabetes (T2D) is one of the major risk factors for cardiovascular disease.

Sodium-glucose co-transporter-2 (SGLT-2) inhibitors have demonstrated powerful action in reducing the risk of heart failure or death from any cardiovascular (CV) cause in subjects with T2D and without diabetes.

The mechanisms of these $\mathrm{CV}$ benefits remain elusive and unexplained.

We hypothesize that SGLT-2 inhibitor treatment will improve both myocardial insulin sensitivity in patients with T2D and coronary artery disease (CAD), and that this improvement may be accompanied, and partly explained, by an increase in myocardial perfusion and/or glucose uptake.

Highly sophisticated and gold standard techniques will be used to assess our hypothesis (euglycemic hyperinsulinemic clamp during fluorodeoxyglucose positron emission tomography/computed tomography (PET/CT) to detect myocardial insulin sensitivity/glucose uptake and PET/CT with ${ }^{13} \mathrm{~N}$-ammonia to assess myocardial perfusion).

\section{DIGITAL FEATURES}

This article is published with digital features, including a summary slide, to facilitate understanding of the article. To view digital features for this article go to https://doi.org/10.6084/ m9.figshare.14561949.

\section{INTRODUCTION}

Insulin resistance appears to be a core factor contributing to impaired myocardial function 
in type 2 diabetes (T2D) $[1,2]$. Myocardial insulin resistance is specifically associated with T2D and is proportional to systemic insulin resistance and to left ventricular systolic dysfunction [3]. In patients with cardiovascular (CV) disease, myocardial segments showing insulin resistance do not return to normal after revascularization, as do myocardial segments with normal insulin sensitivity [4]. In $\mathrm{db} / \mathrm{db}$ mice, which are used to model obesity-induced T2D, with impaired systolic and diastolic dysfunction, the overexpression of the insulinregulated glucose transporter GLUT4 in the myocardium leads to the recovery of normal function [5].

Evidence suggests that reducing substrate availability may have detrimental effects on myocardial function. Myocardial insulin resistance strongly affects myocardial glucose uptake (MGU) [1] and is worsened by circulating levels of free fatty acids (FFA), which are typically high in insulin resistance. However, reducing FFA levels has been reported to suppress cardiac function despite an improvement in insulin sensitivity in healthy subjects [6], and in patients with heart failure $[7,8]$.

In patients with $\mathrm{T} 2 \mathrm{D}$ with and without heart failure, short-term hyperglycemia caused by insulin discontinuation has been reported to have beneficial effects on myocardial function [9]. One possible explanation is that hyperglycemia promotes MGU by mass action, independently of insulin action [10]. Thus, reducing glucose levels in diabetic patients whose plasma glucose levels are chronically high could impair the ability of the heart to preserve contractile efficiency under stress or ischemic conditions. On the other hand, chronically reducing plasma glucose levels and improving insulin sensitivity could decrease gluco- and lipo-toxicity and improve systemic and myocardial glucose metabolism.

The class of drugs known as sodium-glucose cotransporter 2 inhibitors (SGLT-2is) share the same mechanism. They all decrease the renal reabsorption of glucose and induce an increase in glycosuria by inhibiting SGLT-2, the major transporter responsible for renal glucose reabsorption. Some SGLT-2is, such as empagliflozin, dapagliflozin, canagliflozin and ertugliflozin, are currently approved by the U.S. Foods and Drug Administration (FDA) and European Medicines Agency (EMA), while others are still in clinical development. .

Several trials have reported positive outcomes with the administration of SGLT-2is. While the first trial (EMPAREG [11]) demonstrated a significant reduction in CV mortality, this result was not confirmed by the DECLARE trial [12] and only partially supported by the CANVAS trial [13]. It should be pointed out, however, that differences in these study outcomes are often challenging to interpret due to differences in study design, participant characteristics and primary outcomes.

To date, the mechanism(s) responsible for these $\mathrm{CV}$ benefits remain elusive and as yet unexplained [14]. It has been hypothesized that $\mathrm{CV}$ protection is linked to a reduction in heart failure-related deaths (demonstrated by the reduction of hospitalizations for heart failure). Some authors have emphasized the diuretic effect of SGLT-2is, focusing on the reduction of preload and afterload, but the urine volume usually returns to pretreatment levels after a few weeks of treatment with SGLT-2is $[15,16]$ and no randomized trial with diuretics has matched the CV protection obtained with SGLT-2is [17], although a recent trial involving eplerenone in patients with heart failure-but not necessarily diabetes-showed a similar rapid improvement in heart failure outcomes [18]. Other authors have advanced the "myocardial fuel/energetics hypothesis", i.e. the action of SGLT-2is in optimizing cardiac energy metabolism and improving myocardial energetics and substrate efficiency $[19,20]$, However, this hypothesis is not completely unifying or clarifying [21] since ketone body oxidation is already increased in the failing heart [22] and chronic elevations in ketone oxidation may induce functional and structural cardiac maladaptive modifications $[23,24]$, although under conditions of mild but persistent hyperketonemia (as in the case of treatment with SGLT-2 is) b-hydroxybutyrate (b-OHB) is freely taken up by the heart and oxidized in preference to fatty acids. Oxygen consumption is lower when the muscle tries to metabolize ketones instead of FFAs, so this substrate selection (again, during SGLT-2i 
treatment) may improve the transduction of oxygen consumption into more efficient functioning, and therefore contractility, in a highrisk myocardium. Likewise, glucose represents a more efficient fuel when myocardial oxygen delivery is insufficient to fulfill the high demands of fatty acid oxidation. In support of this hypothesis, myocardial infarction seems to be determined by a lack of oxygen rather than substrate availability, and with SGLT-2i treatment myocardial muscle may become more insulin sensitive and thus function more efficiently by metabolizing different substrates and consuming less oxygen (i.e. the quantity of oxygen needed to burn a substrate is more important than the substrate itself).

Insulin resistance is typically associated with a high CV risk since the reduced efficacy on glucose and lipid metabolism often involves the myocardium, in which the coexisting endothelial dysfunction (also directly linked to insulin resistance) may contribute to an alteration in blood flow, as well as in substrate utilization $[25,26]$. In healthy subjects, insulin stimulates myocardial blood flow (MBF) and seems to involve mainly regions of the heart where insulin-mediated MGU is higher, even though this varies among subjects. Similarly, an improvement in myocardial insulin sensitivity may decrease resting MBF with a consequent reduction of $\mathrm{MGU}$, thus raising myocardial metabolism [27]. As already demonstrated, insulin-related coronary blood flow is markedly compromised in persons with T2D compared to healthy subjects $[27,28]$. There seems to be general agreement among the authors of many studies that improvement in insulin resistance and/or changes in substrate utilization and/or modulation of MBF (strictly related to myocardium glucose uptake) may be specific mechanisms (involving the simultaneous modulation of multiple molecular and biochemical pathways, which may not simply or exclusively be related to hyperglycemia) by which SGLT-2is exert their CV benefits [29].

Therefore, a unifying hypothesis is still lacking and, above all, the main action of SGLT2 is in improving cardiac function remains unclear.
Aim

We hypothesize that, by reducing glucotoxicity, dapagliflozin treatment will improve myocardial insulin sensitivity in patients with T2D and coronary artery disease (CAD) and that this improvement may be accompanied, and partly explained, by an increase in myocardial perfusion and/or glucose uptake.

\section{METHODS}

\section{Study Design}

DAPAHEART is a phase III, single-center, randomized, two-arm, parallel-group, doubleblind, placebo-controlled study, conducted in Rome, Italy. It is a proof-of-concept study with the aim to determine the effect of dapagliflozin on myocardial metabolism in subjects with T2D with suboptimal glycemic control on their current anti-hyperglycemic regimen and stable CAD, defined as:

- coronary stenosis $\geq 30 \%$ but $<80 \%$ in at least one native major coronary artery;

- no evidence of critical restenosis, if previously subjected to percutaneous coronary intervention (PCI) as assessed before screening using invasive coronary angiography (ICA) and/or computed tomography angiography (CTA). Furthermore, patients will be screened only if there is evidence of myocardial ischemia in the absence of an indication to revascularization on clinical grounds, as recommended by the European Society of Cardiology (ESC) current guidelines on myocardial revascularization [30].

Patients will be randomized to either dapagliflozin $10 \mathrm{mg}$ once daily or placebo (in addition to Standard of Care).

The study comprises four visits (see Fig. 1); the patient involvement time in the study will be 10 weeks, for a total study duration of 18 months (last patient last visit [LPLV] to clinical study report [CSR]). 


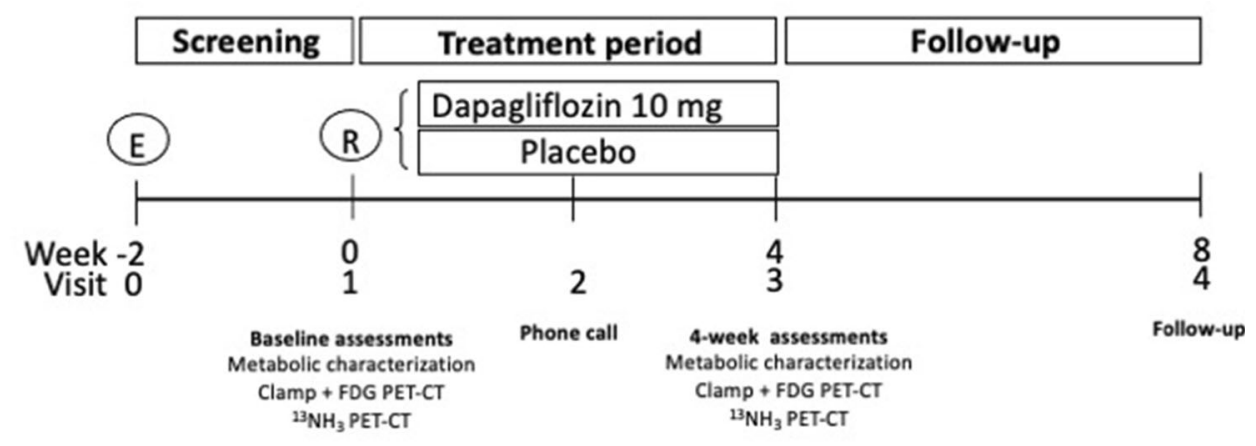

\section{(E): Enrollment}

(R): Randomization

Fig. 1 Study design flowchart. FDG fluorodeoxyglucose, $C T$ computed tomography, $P E T$ positron emission tomography

\section{Sample Selection}

Patients with T2D with CAD not requiring revascularization or clinically stable after PCI with suboptimal glycemic control (glycated hemoglobin [HbA1c] 7-8.5\%, or 58-$69 \mathrm{mmol} / \mathrm{mol}$ ) on their current anti-hyperglycemic regimen will be recruited among patients of the Department of Cardiovascular Medicine, and/or the Center for Endocrine and Metabolic Diseases, at the Fondazione Policlinico Universitario A. Gemelli IRCCS, Università Cattolica del Sacro Cuore, Rome. The experimental sessions will take place in the positron emission tomography/computed tomography (PET/CT) Center.

\section{Ethics Compliance}

The trial protocol has been designed to ensure adherence to Good Clinical Practice guidelines as described in (1) https://www.ema.europa.eu/ en/documents/scientific-guideline/ich-e-6-r2-gui deline-good-clinical-practice-step-5_en.pdf and according to (2) EU Directive 2001/20/EC, 2005/28/EC (https://ec.europa.eu/health/hum an-use/clinical-trials/directive_en); (3) Declaration of Helsinki 1964, and its amendments and subsequent clarification. A copy of the approval will be archived in the study master file in the local study file of the Investigator. The study has also been submitted to and approved by the Hospital/University Ethics Committee (Fondazione Policlinico Universitario A. Gemelli-Università Cattolica del Sacro Cuore. Study Protocol Code GIA-DAP-16-005). In seeking informed consent, the Investigator will explain the nature of the trial, its purpose, the procedures involved, the expected duration, the potential risks and benefits involved and any discomfort it may entail and provide the subject with a copy of the information sheet. The subject will be given sufficient time to consider the trial before deciding whether to participate. Each subject will be informed that participation in the trial is voluntary and that he/she may withdraw from the trial at any time and that withdrawal of consent will not affect his/her subsequent medical treatment or relationship with the treating physician. One of the original copies of the signed consent form will be kept by the Investigator in the study file. The subject will receive the other copy for future reference.

\section{Experimental Plan}

This study consists of a screening phase (Weeks -2 to 0 ), follwed by a 4-week double-blind, placebo-controlled treatment phase and lastly by a 4-week follow-up phase. Patients will be 
randomly assigned to one of the two treatments under blinded conditions according to a centralized procedure. To reduce variability within the treatment groups, the randomization list is stratified by the following factors:

1) patients taking metformin alone;

2) patients taking insulin in addition to any other diabetes therapy;

3) patients taking any other therapy or combination thereof, excluding insulin.

The strata are competitive, therefore each stratum consists of a complete randomization list of 52 numbers. On the basis of the abovementioned factors, the patient is allocated to one of the three strata and randomized in a 1:1 ratio to dapagliflozin or dapagliflozin-matching placebo administered once daily orally for 4 weeks. All subjects receive standard of care for their T2D and CAD. The timing of study assessment is outlined in the study flow chart shown in Fig. 1. Subjects undergo screening assessments during the 2 -week period preceding administration of the first dose of study drug on Day 1.

Throughout the trial the investigators will adjust glucose-lowering therapy at their discretion to achieve the best obtainable glycemic control based on patient conditions (standard of care), with the use of any approved medicine, with the sole exception of SGLT-2is and pioglitazone. Similarly, all the other CV risk factors (including dyslipidemia and hypertension) will be treated according to the best available standard of care.

\section{Study Objectives}

1. The primary objective is to assess the effect of dapagliflozin on myocardial insulin sensitivity (as measured by MGU) in patients with T2D and CAD not requiring revascularization and to test whether the hypothetical improvement is accompanied, or even preceded, by an increase in myocardial perfusion (main secondary outcome).

2. The secondary objective is to assess global heart function, the systemic metabolic effects of dapagliflozin and glycemic control.

\section{Primary Outcome Measure}

1. Effect of dapagliflozin on myocardial insulin sensitivity. MGU (in $\mu \mathrm{mol} / \mathrm{min} / \mathrm{g}$ ) during euglycemic hyperinsulinemic clamp: changes from baseline [time frame: 4 weeks].

\section{Secondary Outcome Measures}

1. Effect on coronary flow reserve (Main secondary outcome). Coronary flow reserve obtained as ratio of myocardial blood flow (mL/g/min) (MBF) during pharmacological stress and MBF at rest by myocardial perfusion PET/CT with ${ }^{13} \mathrm{~N}$-ammonia: changes from baseline [time frame: 4 weeks]

2. Browning of white adipose tissue: changes from baseline. Quantitative measurement of fluorodeoxyglucose (FDG) uptake in pericardial, perirenal, supraclavicular fat by whole-body FDG PET/CT study, expressed as maximum standard uptake value (SUVmax) (changes from baseline) [time frame: 4 weeks].

3. Metabolic systemic effects of dapagliflozin. Whole-body glucose uptake( calculated as $\mathrm{mg} / \mathrm{kg} / \mathrm{min}$ ) during the euglycemic hyperinsulinemic clamp: changes from baseline [time frame: 4 weeks].

4. Effect on left ventricular ejection fraction at rest. Left ventricular ejection fraction (\%) measured by echocardiography and gatedPET with ${ }^{13} \mathrm{~N}$-ammonia at rest: changes from baseline [time frame: 4 weeks].

5. Effect on left ventricular ejection fraction during pharmacological stress. Left ventricular ejection fraction (\%) measured by gated-PET with ${ }^{13} \mathrm{~N}$-ammonia during pharmacological stress: changes from baseline [time frame: 4 weeks].

6. Fasting glucose concentration change from baseline. Measured as fasting glucose concentration $(\mathrm{mg} / \mathrm{dL})$ : changes from baseline [time frame: 4 weeks]. 
7. Glycemic control change from baseline. Measured as HbA1c (\%): changes from baseline [time frame: 4 weeks].

8. Gut microbiota composition change from baseline. Analysis of gut microbiota composition at class, genus, and species levels: changes from baseline [time frame: 4 weeks].

\section{Data Collection and Analysis}

\section{Sample Size}

Sample size calculation was based on published results of a previous trial on the effect of rosiglitazone on mycardial glucose uptake (MGU) in patients with T2D and CAD [31]. Considering an expected delta between the two groups on MGU of $8.7 \mu \mathrm{mol} / 100 \mathrm{~g} / \mathrm{min}$ and a standard deviation of $9.9,23$ patients per treatment group are considered a sufficient number to reject the null hypothesis that the population means of the two groups are equal with a power of $80 \%$ and an alpha of 0.05 . Assuming a $10 \%$ loss in each group due to protocol violations/loss to follow-up, we estimate that the total number should be 26 patients per group.

\section{Randomization and treatment allocation}

Patients will be assigned to the study treatment on a 1:1 ratio, on the basis of a stratified randomization list generated by an independent statistician who will maintain it for the entire duration of the study. The randomization list will be stratified by the three abovementioned factors. The strata will be competitive; therefore, each stratum will consist of a complete randomization list of 52 numbers. The patient will be allocated to one of the three strata and randomized in a $1: 1$ ratio to dapagliflozin or dapagliflozin-matching placebo administered once daily orally for 4 weeks. The three lists, one for each stratum, will be managed centrally, as will the randomization procedure.

\section{Treatment comparisons}

Demographic and baseline characteristics (e.g. gender, age, racial or ethnic origin, height and weight, body mass index (BMI), blood pressure and other characteristics) will be summarized for each treatment group. In addition, smoking and alcohol habits, diabetic and cardiovascular medical history, baseline laboratory results and prior medications will be summarized by treatment group. Binary and ordinal characteristics will be summarized by counts and percentages, while continuous variables will be represented by means and standard deviations or medians and percentiles, as appropriate. Any variables with treatment imbalances may be considered as covariates for further analysis of an exploratory nature. Such covariates will be identified on the basis of the clinical relevance of the observed treatment difference.

\section{Primary comparison of interest}

Change from baseline to 4 weeks in insulinmediated MGU is the primary endpoint of the study.

Analysis of covariance (ANCOVA) will be performed in order to calculate the statistical power. Data will be examined for normal distribution. Parametric and/or non-parametric tests will be used, as appropriate. Within-group differences will be assessed using the $t$-test (or equivalent non-parametric test) for paired data; between-group differences will be assessed using the $t$ test (or equivalent non-parametric test) for unpaired data. In addition, tests for repeated measurements will be used to account for treatment versus group effects and interactions.

Two interim analyses will be preprogrammed at the enrollment of 30 and $60 \%$ of the patients, respectively. A stopping rule will be implemented to halt enrollment if either the primary or the main secondary outcome of interest differs statistically between treatment groups. In this case, a Bonferroni-adjusted $p$ value of 0.016 will be implemented (to account for the two interim analyses plus the final analysis, e.g. $0.05 / 3)$.

For inclusion/exclusion criteria and study procedure, please refer to the Electronic Supplementary Material files. 


\section{STRENGTHS AND LIMITATIONS}

The main strengths of our study are:

- highly sophisticated and gold standard methods to assess insulin sensitivity (euglycemic hyperinsulinemic clamp), myocardial and whole-body metabolism (glucose uptake calculated during FDG PET/CT study and the euglycemic hyperinsulinemic clamp), myocardial blood flow and coronary reserve (by PET/CT with ${ }^{13} \mathrm{~N}$-ammonia) and browning effect of the study drug (by wholebody FDG PET/CT study);

- highly selected study population: patients with T2D with a narrow HbA1c range (HbA1c 7-8.5\%) and CAD not requiring revascularization or clinically stable after PCI;

- the combination of primary and main secondary outcomes will allow us to elucidate the CV benefit of SGLT-2is.

Our study has some limitations.

- The inclusion criteria reduce the number of eligible patients; furthermore, the methods are highly time- and cost-consuming, thus limiting the number of patients that can be enrolled.

- The period of treatment appears to be sufficient to determine the effect of dapagliflozin on myocardial metabolism, since metabolic control is not a primary endpoint of the study; still, being a pilot study, myocardial adaptation to removal of insulin resistance could require a different time interval.

\section{DISCUSSION}

Type 2 diabetes is one of the major risk factors for cardiovascular disease (CVD) [32] and overall mortality [33] despite advances in treatment. In 2008, the FDA issued specific guidelines regarding the need for anti-hyperglycemic therapies to be evaluated for CV safety in largescale randomized trials. The EMPA-REG OUTCOME trial, the first $\mathrm{CV}$ end-point trial for glucose-lowering agents [34], assessed the SGLT2 inhibitor empagliflozing at 10 or $25 \mathrm{mg}$ /day versus placebo in patients with T2D with established atherosclerotic CVD and reported a $14 \%$ reduction in major CV events and marked relative risk reductions in CV mortality (38\%), hospitalization for heart failure (35\%), and death from any cause (32\%), within a short follow-up period [11].

Similarly, the Canagliflozin CV Assessment Study (CANVAS) showed that canagliflozin decreased classical 3-point major adverse coronary events (MACE), i.e. CV death, non-fatal myocardial infarction and non-fatal stroke, by $14 \%$ (hazard ratio $0.86,95 \%$ confidence interval 0.75-0.97) [13].

In a large $\mathrm{T} 2 \mathrm{D}$ population of more than 40,000 T2D patients, another SGLT-2i, dapagliflozin, was associated with $21 \%$ lower risk of MACE, 54\% lower all-cause mortality risk and $38 \%$ lower risk of hospitalization for heart failure compared to other oral anti-diabetic drugs (OADs) [13]. SGLT-2is have also demonstrated powerful action in reducing the risk for heart failure or death from any CV causes in subjects without diabetes [35]. Even though different in terms of population, study design and endpoints, all cardiovascular outcome trials with SGLT-2i demonstrated CV protection and reduction in heart failure hospitalizations, especially in patients with reduced ejection fraction [36-38].

As expected, the prompt and substantial cardioprotective effects of SGLT-2is have generated investigation into the mechanism(s) responsible for these benefits, as yet not fully elucidated.

Treatment with SGLT-2is induces an increase in FFA oxidation, which in turn increases ketogenesis. This process is the basis for the "Thrifty Substrate" hypothesis proposed by Ferrannini et al. [19], who suggest that treatment with empagliflozin (and, in the light of available data in literature we can expand the discussion to the entire class of SGLT-2is) enhances cardiac energy metabolism, improving myocardial energetics and substrate efficiency by increasing the supply of b-OHB to the high risk heart. b-OHB is a "super fuel" and could represent a better substrate in terms of energy production than fatty acids and glucose in a "damaged" heart. The selection of b-OHB instead of fatty 
acids by the heart may enhance efficiency at the mitochondrial level and may be responsible for the improved myocardial performance.

Lopashuck and Verma [21], however, have identified some limitations to this theory. Starting from the demonstration of the already upregulated ketone body oxidation in the damaged heart, they affirm that a chronic elevation in ketone oxidation could induce maladaptive modifications. Ketone oxidation may also lead to a depletion of tricarboxylic acid (TCA) cycle intermediates and subsequently to a reduction of mitochondrial function by decreasing oxidative phosphorylation. Furthermore, although it is already clear that SGLT-2i treatment leads to an increase in ketone synthesis, we cannot exclude a concomitant modification in the entire ketone clearance, although whether the increase of ketone bodies is due to increased production (induced by SGLT-2 inhibition) or to less catabolism, i.e. reduction of clearance, has not yet been clarified.

The changes in metabolic profile in the myocardium with chronic hyperglycemia are already known and entail a decrease in glucose uptake and glucose oxidation and an increase in fatty acid oxidation [39]. In addition, insulin resistance per se contributes to impaired myocardial function in diabetes since the heart also becomes insulin resistant [40].

Starting from the latter point, Merovci et al. [41] demonstrated that lowering plasma glucose concentration by inducing glucosuria in individuals with T2D significantly improves insulinstimulated tissue glucose availability. Both insulin-stimulated whole-body glucose availability and tissue glucose availability significantly improved after the reduction in blood glucose levels determined by dapagliflozin treatment, providing strong evidence for the glucotoxicity hypothesis [42] in humans. Conversely, it has been shown that SGLT-2is do not promote any improvement in myocardial and peripheral insulin sensitivity [43]. However, the patients enrolled in this trial already had optimal glucose control at randomization (6.8 and $7.0 \%$ in placebo and treatment group, respectively). While these results suggest that the use of an SGLT-2i does not improve glucose metabolism when HbA1c is already at target (though a decrease in visceral adipose volume has been described after just 8 weeks of treatment), they also confirm our working hypothesis, which is based on changes in glucose and FFA metabolism. In a recent paper, Lauritsen et al. [44] describe MGU reduction after SGLT-2i treatment (empagliflozin) with a parallel reduction in resting MBF. A potential explanation for these results could be a reduction in insulin concentration, since it has already been demonstrated that hyperinsulinemia induces an expected increase in MGU with a significant increase in resting myocardial blood flow [27]. Unfortunately, Lauritsen et al. [44] did not measure insulin or perform any euglycemic hyperinsulinemic clamp, which would have been important to confirm the latter hypothesis. Similarly, Oldgren et al. also describe a decrease in MBF after 6 weeks of dapagliflozin treatment, but in this case it was not significant [45]. All these data suggest that the amelioration of insulin resistance, dependent on blood flow induced by SGLT-2is, could be one of the intriguing hypotheses explaining the CV benefits of these drugs [46]. Indeed, in the heart, insulin stimulates MBF in healthy subjects, and this action seems to be enhanced in regions of the heart where insulin-mediated MGU is higher [27]. In T2D, however, the insulin-related coronary blood flow is markedly compromised [28].

Moving from the molecular level to the systemic level, it has already been shown that poor glucose control (generally evaluated by HbA1c) is closely linked to an increase in $\mathrm{CV}$ risk, including ischemic heart disease and heart failure [46-48]. However, intensive glucose control trials have failed to demonstrate a reduction in the development of CV-related or all-cause heart failure [49-51].

In hyperglycemic conditions, the high level of glucose increases peripheral tissue glucose uptake, without a consequent increase in the production of ATP. In fact, in the heart, the production of ATP is more important than the availability and/or source of energy production. The improvement of myocardial insulin sensitivity could result in more efficient ATP 
production [52], even with a reduced glucose uptake [53].

With this study we aim to demonstrate that treatment with dapagliflozin is associated with an improvement in myocardial insulin sensitivity (as indicated by measures of MGU and/or MBF) in patients with T2D, and to determine whether this improvement can be attributed to an amelioration of whole-body (and tissuespecific) insulin resistance, to increased myocardial perfusion and/or glucose uptake or to "super fuel" availability. We will determine whether there is a distinct degree of improvement in myocardial-wall conditions subtended by affected versus non-affected coronary vessels, and if this relates to changes in left ventricular function.

\section{ACKNOWLEDGEMENTS}

Medical writing and editorial assistance. We thank Serena Rotunno (Università Cattolica del Sacro Cuore) for editorial assistance in the writing of this article. This assistance was funded by the authors.

Authorship. All authors meet the International Committee of Medical Journal Editors (ICMJE) criteria for authorship for this article, take responsibility for the integrity of the work as a whole, and have given their approval for this version to be published.

Author contributions. GPS, PI and AG designed the trial. MAG, LL, ML and AG described and reviewed the nuclear procedures. DD and FC described and reviewed the cardiological tests. PMF designed the statistical plan and led the independent interim analysis. FC, $\mathrm{TM}, \mathrm{CC}$ and UC described the metabolic procedures. GPS, FC and LL gave the major contribution in writing the manuscript. All authors have read and approved the final manuscript.

Disclosures. Gian Pio Sorice, Francesca Cinti, Lucia Leccisotti, Domenico D'Amario, Margherita Lorusso, Maria Angela Guzzardi, Teresa Mezza, Camilla Cocchi, Umberto
Capece, Pietro Manuel Ferraro, Filippo Crea, Alessandro Giordano, Patricia Iozzo and Andrea Giaccari have nothing to disclose.

Funding. The submitted project was funded by a pharmaceutical company (AstraZeneca) to cover tracer costs. The authors supported the journal's Rapid Service fee.

Compliance with ethics guidelines. The trial protocol has been designed to ensure adherence to Good Clinical Practice guidelines as described in (1) https://www.ema.europa.eu/en/ documents/scientific-guideline/ich-e-6-r2-guide line-good-clinical-practice-step-5_en.pdf and according to (2) EU Directive 2001/20/EC, 2005/28/EC (https://ec.europa.eu/health/hu man-use/clinical-trials/directive_en); (3) Declaration of Helsinki 1964, and its amendments and subsequent clarification. A copy of the approval will be archived in the study master file in the local study file of the Investigator. The study has also been submitted to and approved by the Hospital/University Ethics Committee (Fondazione Policlinico Universitario A. Gemelli-Università Cattolica del Sacro Cuore. Study Protocol Code GIA-DAP-16-005). In seeking informed consent, the Investigator will explain the nature of the trial, its purpose, the procedures involved, the expected duration, the potential risks and benefits involved and any discomfort it may entail and provide the subject with a copy of the information sheet. The subject will be given sufficient time to consider the trial before deciding whether to participate. Each subject will be informed that participation in the trial is voluntary and that he/she may withdraw from the trial at any time and that withdrawal of consent will not affect his/her subsequent medical treatment or relationship with the treating physician. One of the original copies of the signed consent form will be kept by the Investigator in the study file. The subject will receive the other copy for future reference.

Data availability. Data sharing is not applicable to this article as no datasets were generated or analyzed during the current study. 
Open Access. This article is licensed under a Creative Commons Attribution-NonCommercial 4.0 International License, which permits any non-commercial use, sharing, adaptation, distribution and reproduction in any medium or format, as long as you give appropriate credit to the original author(s) and the source, provide a link to the Creative Commons licence, and indicate if changes were made. The images or other third party material in this article are included in the article's Creative Commons licence, unless indicated otherwise in a credit line to the material. If material is not included in the article's Creative Commons licence and your intended use is not permitted by statutory regulation or exceeds the permitted use, you will need to obtain permission directly from the copyright holder. To view a copy of this licence, visit http://creativecommons.org/licenses/by$\mathrm{nc} / 4.0 /$.

\section{REFERENCES}

1. Kasznicki J, Drzewoski J. Heart failure in the diabetic population-pathophysiology, diagnosis and management. Arch Med Sci. 2014;10:546-56.

2. Masi S, Lautamaki R, Guiducci L, et al. Similar patterns of myocardial metabolism and perfusion in patients with type 2 diabetes and heart disease of ischaemic and non-ischaemic origin. Diabetologia. 2012;55:2494-500.

3. Iozzo P, Chareonthaitawee P, Dutka D, Betteridge DJ, Ferrannini E, Camici PG. Independent association of type 2 diabetes and coronary artery disease with myocardial insulin resistance. Diabetes. 2002;51:3020-4.

4. Marinho NV, Keogh BE, Costa DC, Lammerstma AA, Ell PJ, Camici PG. Pathophysiology of chronic left ventricular dysfunction. New insights from the measurement of absolute myocardial blood flow and glucose utilization. Circulation. 1996;93: 737-44.

5. Semeniuk LM, Kryski AJ, Severson DL. Echocardiographic assessment of cardiac function in diabetic $\mathrm{db} / \mathrm{db}$ and transgenic db/db-hGLUT4 mice. Am J Physiol Heart Circ Physiol. 2002;283:H976-982.

6. Lehto HR, Parkka J, Borra R, et al. Effects of acute and one-week fatty acid lowering on cardiac function and insulin sensitivity in relation with myocardial and muscle fat and adiponectin levels. J Clin Endocrinol Metab. 2012;97:3277-84.

7. Salerno A, Fragasso G, Esposito A, et al. Effects of short-term manipulation of serum FFA concentrations on left ventricular energy metabolism and function in patients with heart failure: no association with circulating bio-markers of inflammation. Acta Diabetol. 2015;52:753-61.

8. Tuunanen H, Engblom E, Naum A, et al. Free fatty acid depletion acutely decreases cardiac work and efficiency in cardiomyopathic heart failure. Circulation. 2006;114:2130-7.

9. Nielsen R, Norrelund H, Kampmann U, Botker HE, Moller N, Wiggers $\mathrm{H}$. Effect of acute hyperglycemia on left ventricular contractile function in diabetic patients with and without heart failure: two randomized cross-over studies. PLoS ONE. 2013;8: e53247.

10. Guzzardi MA, Hodson L, Guiducci L, et al. Independent effects of circulating glucose, insulin and NEFA on cardiac triacylglycerol accumulation and myocardial insulin resistance in a swine model. Diabetologia. 2014;57:1937-46.

11. Zinman B, Wanner C, Lachin JM, et al. Investigators E-RO: empagliflozin, cardiovascular outcomes, and mortality in type 2 diabetes. $\mathrm{N}$ Engl J Med. 2015;373:2117-28.

12. Wiviott SD, Raz I, Bonaca MP, et al. Dapagliflozin and cardiovascular outcomes in type 2 diabetes. N Engl J Med. 2019;380:347-57.

13. Neal B, Perkovic V, Matthews DR. Canagliflozin and cardiovascular and renal events in type 2 diabetes. N Engl J Med. 2017;377:2099.

14. Sattar N, McLaren J, Kristensen SL, Preiss D, McMurray JJ. SGLT2 Inhibition and cardiovascular events: why did EMPA-REG Outcomes surprise and what were the likely mechanisms? Diabetologia. 2016;59:1333-9.

15. Tikkanen I, Narko K, Zeller C, et al. Empagliflozin reduces blood pressure in patients with type 2 diabetes and hypertension. Diabetes Care. 2015;38: 420-8.

16. Sha S, Polidori D, Heise T, et al. Effect of the sodium glucose co-transporter 2 inhibitor canagliflozin on plasma volume in patients with type 2 diabetes mellitus. Diabetes Obes Metab. 2014;16:1087-95.

17. ALLHAT Officers and Coordinators for the ALLHAT Collaborative Research Group. Major outcomes in high-risk hypertensive patients randomized to angiotensin-converting enzyme inhibitor or calcium channel blocker vs diuretic: the 
Antihypertensive and Lipid-Lowering Treatment to Prevent Heart Attack Trial (ALLHAT). JAMA. 2002;288:2981-97.

18. Zannad F, McMurray JJ, Krum H, et al. Eplerenone in patients with systolic heart failure and mild symptoms. N Engl J Med. 2011;364:11-21.

19. Ferrannini E, Mark M, Mayoux E. CV Protection in the EMPA-REG OUTCOME Trial: a "Thrifty Substrate" Hypothesis. Diabetes Care. 2016;39: 1108-14.

20. Mudaliar S, Alloju S, Henry RR. Can a shift in fuel energetics explain the beneficial cardiorenal outcomes in the EMPA-REG OUTCOME study? A Unifying Hypothesis. Diabetes Care. 2016;39:1115-22.

21. Lopaschuk GD, Verma S. Empagliflozin's fuel hypothesis: not so soon. Cell Metab. 2016;24: 200-2.

22. Aubert G, Martin OJ, Horton JL, et al. The failing heart relies on ketone bodies as a fuel. Circulation. 2016;133:698-705.

23. Horton JL, Martin OJ, Lai L, et al. Mitochondrial protein hyperacetylation in the failing heart. JCI Insight 2016;2(1):e84897.

24. Russell RR 3rd, Taegtmeyer H. Changes in citric acid cycle flux and anaplerosis antedate the functional decline in isolated rat hearts utilizing acetoacetate. J Clin Invest. 1991;87:384-90.

25. DeFronzo RA. Insulin resistance: a multifaceted syndrome responsible for NIDDM, obesity, hypertension, dyslipidaemia and atherosclerosis. Neth J Med. 1997;50:191-7.

26. Playford D, Watts GF. Endothelial dysfunction, insulin resistance and diabetes: exploring the web of causality. Aust N Z J Med. 1999;29:523-34.

27. Iozzo P, Chareonthaitawee P, Di Terlizzi M, Betteridge DJ, Ferrannini E, Camici PG. Regional myocardial blood flow and glucose utilization during fasting and physiological hyperinsulinemia in humans. Am J Physiol Endocrinol Metab. 2002;282: E1163-1171.

28. Jagasia D, Whiting JM, Concato J, Pfau S, McNulty PH. Effect of non-insulin-dependent diabetes mellitus on myocardial insulin responsiveness in patients with ischemic heart disease. Circulation. 2001;103:1734-9.

29. Vettor R, Inzucchi SE, Fioretto P. The cardiovascular benefits of empagliflozin: SGLT2-dependent and independent effects. Diabetologia. 2017;60:395-8.
30. Neumann FJ, Sousa-Uva M, Ahlsson A, et al. 2018 ESC/EACTS Guidelines on myocardial revascularization. Eur Heart J. 2019;40:87-165.

31. Lautamaki R, Airaksinen KE, Seppanen M, et al. Rosiglitazone improves myocardial glucose uptake in patients with type 2 diabetes and coronary artery disease: a 16-week randomized, double-blind, placebo-controlled study. Diabetes. 2005;54:2787-94.

32. Emerging Risk Factors Collaboration; Sarwar N, Gao $P$, et al.. Diabetes mellitus, fasting blood glucose concentration, and risk of vascular disease: a collaborative meta-analysis of 102 prospective studies. Lancet. 2010;375:2215-22.

33. IDF Diabetes Atlas Group. Update of mortality attributable to diabetes for the IDF Diabetes atlas: estimates for the year 2011. Diabetes Res Clin Pract 2013;100:277-9.

34. Ferrannini E, DeFronzo RA. Impact of glucose-lowering drugs on cardiovascular disease in type 2 diabetes. Eur Heart J. 2015;36:2288-96.

35. McMurray JJV, Solomon SD, Inzucchi SE, et al. Dapagliflozin in patients with heart failure and reduced ejection fraction. N Engl J Med. 2019;381: 1995-2008.

36. Packer M, Anker SD, Butler J, et al. Cardiovascular and renal outcomes with empagliflozin in heart failure. N Engl J Med. 2020;383:1413-24.

37. Bhatt DL, Szarek M, Steg PG, et al. Sotagliflozin in patients with diabetes and recent worsening heart failure. N Engl J Med. 2021;384:117-28.

38. Cannon CP, Pratley R, Dagogo-Jack S, et al. Cardiovascular outcomes with ertugliflozin in type 2 diabetes. N Engl J Med. 2020;383:1425-35.

39. Lopaschuk GD, Ussher JR, Folmes CD, Jaswal JS, Stanley WC. Myocardial fatty acid metabolism in health and disease. Physiol Rev. 2010;90:207-58.

40. Zhang L, Jaswal JS, Ussher JR, et al. Cardiac insulinresistance and decreased mitochondrial energy production precede the development of systolic heart failure after pressure-overload hypertrophy. Circ Heart Fail. 2013;6:1039-48.

41. Merovci A, Mari A, Solis-Herrera C, et al. Dapagliflozin lowers plasma glucose concentration and improves beta-cell function. J Clin Endocrinol Metab. 2015;100:1927-32.

42. Rossetti L, Giaccari A, DeFronzo RA. Glucose toxicity. Diabetes Care. 1990;13:610-30.

43. Latva-Rasku A, Honka MJ, Kullberg J, et al. The SGLT2 inhibitor dapagliflozin reduces liver fat but 
does not affect tissue insulin sensitivity: a randomized, double-blind, placebo-controlled study with 8 -week treatment in type 2 diabetes patients. Diabetes Care. 2019;42:931-7.

44. Lauritsen KM, Nielsen BRR, Tolbod LP, et al. SGLT2 inhibition does not affect myocardial fatty acid oxidation or uptake, but reduces myocardial glucose uptake and blood flow in individuals with type 2 diabetes: a randomized double-blind placebocontrolled crossover trial. Diabetes. 2021;70:800-8.

45. Oldgren J, Laurila S, Akerblom A, et al. Effects of 6 weeks of treatment with dapagliflozin, a sodiumglucose co-transporter-2 inhibitor, on myocardial function and metabolism in patients with type 2 diabetes: a randomized, placebo-controlled, exploratory study. Diabetes Obes Metab. 2021. https://doi.org/10.1111/dom.14363.

46. Giaccari A. Sodium-glucose co-transporter inhibitors: medications that mimic fasting for cardiovascular prevention. Diabetes Obes Metab. 2019;21: 2211-8

47. Haffner SM, Lehto S, Ronnemaa T, Pyorala K, Laakso M. Mortality from coronary heart disease in subjects with type 2 diabetes and in nondiabetic subjects with and without prior myocardial infarction. N Engl J Med. 1998;339:229-34.

48. Iribarren C, Karter AJ, Go AS, et al. Glycemic control and heart failure among adult patients with diabetes. Circulation. 2001;103:2668-73.

49. Turner RC, Millns H, Neil HA, et al. Risk factors for coronary artery disease in non-insulin dependent diabetes mellitus: United Kingdom Prospective Diabetes Study (UKPDS: 23). BMJ. 1998;316:823-8.

50. ACCORD Study Group; Cushman WC, Evans GW, et al. Effects of intensive blood-pressure control in type 2 diabetes mellitus. N Engl J Med. 2010;362: 1575-85.

51. ADVANCE Collaborative Group; Patel A, MacMahon $\mathrm{S}$, et al. Intensive blood glucose control and vascular outcomes in patients with type 2 diabetes. N Engl J Med. 2008;358:2560-72.

52. Duckworth W, Abraira C, Moritz T, et al. Glucose control and vascular complications in veterans with type 2 diabetes. N Engl J Med. 2009;360:129-39.

53. Riehle C, Abel ED. Insulin signaling and heart failure. Circ Res. 2016;118:1151-69. 